\title{
Shape-Shifting Proteins Follow Diffusion Rules
}

\author{
How quickly a protein diffuses in a liquid depends directly on its radius, \\ which changes as the protein's conformation fluctuates.
}

\section{By Rachel Berkowitz}

$\square$

roteins are constantly morphing shape. These changes, which can be caused by temperature shifts, for example, alter the protein's radius, affecting its ability to move through a cell membrane. Using molecular dynamics simulations, Eiji Yamamoto of Keio University, Japan, and colleagues now show that radius changes can also directly impact a protein's "instantaneous" diffusivity, which is its diffusivity at any moment in time [1]. The finding could help explain recent experimental measurements of the randomly fluctuating diffusivities of tracer particles, such as colloids, quantum dots, or fluorescently labeled proteins in supercooled liquids, soft materials, and biological systems.

A tracer travels in a liquid by colliding with and bouncing off liquid molecules. The tracer's diffusivity is traditionally determined by its radius and by the liquid's viscosity. But in some systems, such as polymers in dilute solutions, where both the liquid's viscosity and the tracer's radius are assumed to remain constant, the diffusivity has been observed to fluctuate

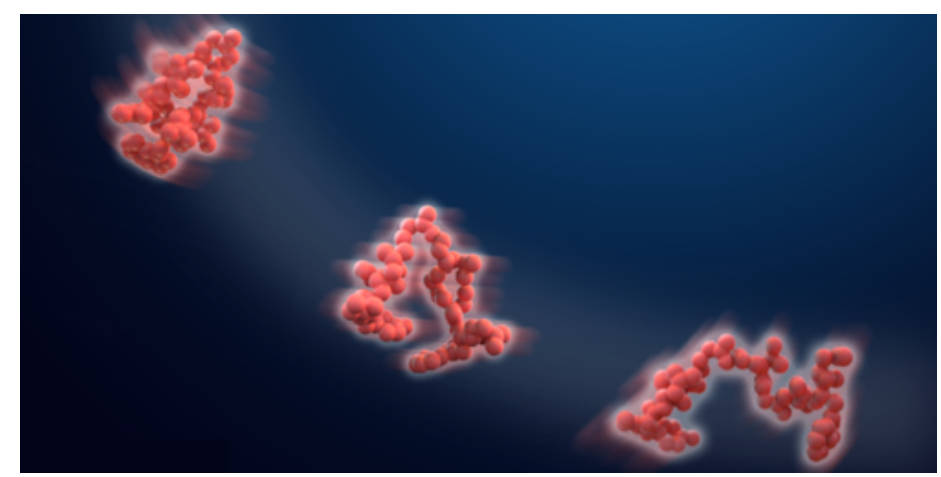

Credit: Mayu S. Terakawa/Kyoto University over time.

To explore the origin of these fluctuations, Yamamoto and his team simulated the motion of proteins suspended in water under different pressure and temperature conditions. From these simulations they calculated the time-dependent radius and diffusivity of each protein.

The team found that when the conditions caused an increase in the protein's radius, the measured diffusivity decreased, and vice versa. They derived a relationship between radius and diffusivity, showing that it follows a time-dependent version of the classical diffusivity relationship for a spherical particle. The team speculates that the relationship will hold for more complex proteins than the ones they studied, for other tracers found in cells, and for other deformable macromolecules and polymers.

Rachel Berkowitz is a Corresponding Editor for Physics based in Vancouver, Canada.

\section{REFERENCES}

1. E. Yamamoto et al., "Universal relation between instantaneous diffusivity and radius of gyration of proteins in aqueous solution," Phys. Rev. Lett. 126, 128101 (2021). 